To appear in Proc. 3rd Conference on Computer Supported Collaborative Learning, Stanford, December 11-15, 1999.

\title{
Effects of Alternate Representations of Evidential Relations on Collaborative Learning Discourse
}

\author{
Daniel D. Suthers
}

University of Hawai'i at Manoa, Department of Information and Computer Sciences

\begin{abstract}
Over the past decade or so, a number of software environments have been created to support students engaged in collaborative investigations in science (e.g., Belvedere, CoVis, CSILE, SenseMaker, and WebCamile). These environments have used a variety of representations for recording information such as alternate hypotheses, empirical observations, and evidential relations (e.g., node-link graphs, structured lists, and containers). There are both empirical and theoretical reasons to believe that the expressive constraints imposed by a representation and the information (or lack of information) that a representation makes salient may have important effects on students' discourse during collaborative learning. However, to date no systematic study has been undertaken to explore possible effects. This paper outlines a research agenda to address this need; provides theoretically motivated predictions; and reports initial results from a pilot study. Students worked together in groups of two on hypertext-based "science challenge" problems. Two groups used each of free text (MS Word), matrix (Excel) or graph (Belvedere) representations of evidence, for a total of six groups. Analysis of discourse transcripts suggests that these representations have quite different effects on the extent to which students discuss evidential relations.
\end{abstract}

Keywords: HCI, discourse analysis, representational tools

\section{Introduction}

For a number of years, the author and his colleagues (see acknowledgments) have been building, testing, and refining a diagrammatic environment ("Belvedere") intended to support secondary school children's learning of critical inquiry skills in the context of science. The diagrams were first designed to capture scientific argumentation, and later simplified to focus on evidential relations between data and hypotheses. This change was driven in part by a refocus on collaborative learning, which led to a major change in how we viewed the role of the interface representations. Rather than being a medium of communication or a formal record of the argumentation process, we came to view the representations as resources (stimuli and guides) for conversation (Roschelle, 1994; Suthers, 1995).

Meanwhile, various projects with similar goals (i.e., critical inquiry in a collaborative learning context) were using radically different representational systems (Bell, 1997; Guzdial et al., 1997; O'Neill \& Gomez, 1994; Scardamalia et al., 1992; Suthers et al., 1997). There are both empirical and theoretical reasons, some of which are summarized in this paper, to believe that the expressive constraints imposed by a representation and the information (or lack of information) that a representation makes salient may have important effects on students' discourse during 
To appear in Proc. 3rd Conference on Computer Supported Collaborative Learning, Stanford, December 11-15, 1999.

collaborative learning. However, to date no systematic study has been undertaken to explore possible effects of this variable on collaborative learning. This paper motivates and describes such a study being undertaken by the author and reports initial results from a pilot study.

\section{Representations in critical inquiry software}

To provide examples and motivation for discussion, several alternate representational approaches taken in computer supported collaborative learning (CSCL) systems for critical inquiry are characterized below.

Hypertext/hypermedia systems include CLARE (Wan \& Johnson, 1994), CSILE (Scardamalia et al., 1992), the Collaboratory Notebook (O’Neill \& Gomez, 1994), and Web-Camile and WebSMILE (Guzdial et al., 1997). (Seminal systems include gIBIS (Conklin \& Begeman, 1987) and NoteCards (Harp \& Neches, 1988), which were not developed for educational applications.) These systems all have in common a linking of different comments relevant to an issue, usually with categorization of the hyperlinks or their targets with labels. There is wide variation in this category: some take the form of a threaded discussion or other tree structure that may be viewed in summary form (see Figure 1a for a characterization), while others support construction of graphs of "nodes" or "cards" through which one navigates, viewing one card at a time. Mature systems such as CSILE or its successor, Knowledge Forum, use several of the representational approaches discussed herein.

Several argument mapping environments, including Belvedere (Suthers et al., 1997; Suthers \& Weiner, 1995), ConvinceMe (Ranney et al., 1995), and Euclid (Smolensky et al., 1987), utilize node-link graphs representing rhetorical, logical, or evidential relationships between assertions (usually categorized as "hypothesis" versus "data" or "evidence"). Belvedere is characterized in Figure 1c: rounded shapes represent hypotheses and rectangles represent empirical observations. The entire graph is viewed and manipulated at once, distinguishing these systems from hypermedia environments in which one normally works with one node of the graph at a time.

SenseMaker (Bell, 1997) exemplifies an intermediate approach between graphs and hierarchies. Statements are organized in a 2-dimensional space and viewed all at once, as in argument graphs (see Figure 1b). However, SenseMaker uses containment rather than links to represent the relationship of evidential support: an empirical statement is placed inside the box of the theory it supports. SenseMaker also uses containment to represent decomposition of a theory into hypotheses, a feature that was tried in early versions of Belvedere.

Finally, another representation is an evidence or criteria matrix. Several forms are possible. One organizes hypotheses along one axis, and empirical evidence along another, with matches between the two being expressed symbolically in the cells of the matrix (e.g., Figure 2c). Puntambekar et al. (1997) experimented with a matrix representation in a paper-based collaboration tool.

The differences in representational notations provided by existing software for critical inquiry is striking. Yet more striking is the fact that there appear to be no systematic studies comparing the 
To appear in Proc. 3rd Conference on Computer Supported Collaborative Learning, Stanford, December 11-15, 1999.

effects of external representations on collaborative learning discourse. Exceptions include (Guzdial, 1997; Wojahn et al., 1998). Given that these representations define the fundamental character of software intended to guide learning, a systematic comparison is overdue.

Substantial research has been conducted concerning the role of external representations in individual problem solving, generally showing that the kind of external representation used to depict a problem may determine the ease with which the problem is solved (Koedinger, 1991; Kotovsky \& Simon, 1990; Larkin \& Simon, 1987; McGuiness, 1986; Zhang, 1997). One might ask whether this research is sufficient to predict the effects of representations in collaborative learning. A related but distinct line of work undertaken in collaborative learning contexts is needed for several reasons. The interaction of the cognitive processes of several agents is different than the reasoning of a single agent (Okada \& Simon, 1997; Perkins, 1993), so may be affected by external representations in different ways. In particular, shared external representations can be used to coordinate distributed work, and will serve this function different ways according to their representational biases. Also, the mere presence of representations in a shared context with collaborating agents may change each individual's cognitive processes. One person can ignore discrepancies between thought and external representations, but an individual working in a group must constantly refer back to the shared external representation while coordinating activities with others. Thus it is conceivable that external representations have a greater effect on individual cognition in a social context than they do when working alone (Micki Chi, personal communication). Finally, much prior work on the role of external representations in individual problem solving has used well-defined problems. Further study is needed on ill structured, open-ended problems such as those typical of scientific inquiry.

\section{Representational bias}

This section sketches a theoretical perspective to guide the research agenda, beginning with definitions. Representational tools are artifacts (such as software) with which users construct, examine, and manipulate external representations of their knowledge. The present work is concerned with symbolic as opposed to analogical representations. A representational tool is an implementation of a representational notation that provides a set of primitive elements out of which representations can be constructed. Developers choose a representational notation and instantiate it as a representational tool, while the user of the tool constructs particular representational artifacts in the tool. The present work focuses on interactions between learners and other learners, specifically verbal and gestural interactions termed collaborative learning discourse.

Each given representational notation manifests a particular representational bias, expressing certain aspects of one's knowledge better than others (Utgoff, 1986). The phrase knowledge unit is used to refer generically to components of knowledge one might wish to represent, such as hypotheses, statements of fact, concepts, relationships, rules, etc. Representational bias manifests in two major ways: Constraints: limits on logical expressiveness, and in the sequence in which knowledge units can be expressed (Reader, unpublished, Stenning \& Oberlander, 1995); and Salience: how the representation facilitates processing of certain knowledge units, possibly at the expense of others (Larkin \& Simon, 1987). Representational tools mediate collaborative learning 
To appear in Proc. 3rd Conference on Computer Supported Collaborative Learning, Stanford, December 11-15, 1999.

discourse by providing learners with the means to articulate emerging knowledge in a persistent medium, inspectable by all participants, where the knowledge then becomes part of the shared context. Representational bias constrains the knowledge that can be expressed in the shared context, and makes some of that knowledge more salient and hence a likely topic of discussion. Sources of constraint and salience are discussed below.

Zhang (1997) distinguishes cognitive and perceptual operators in reasoning with representations. Cognitive operations operate on internal representations; while perceptual operations operate on external representations. Perceptual operations take place without making an internal copy of the representation, although internal representations may change as a result of these operations. Expressed in terms of Zhang's framework, the present work is concerned primarily with perceptual operations on external representations: the question is how representations that reside in learners' perceptually shared context mediate collaborative learning discourse. While it is the case that cognitive operations on internal representations will influence interactions in the social realm, CSCL system builders do not design internal representations - they design tools for constructing external representations.

Stenning and Oberlander (1995) distinguish constraints inherent in the logical properties of a representational notation from constraints arising from the architecture of the agent using the representational notation. This corresponds roughly to the present author's distinction between "constraints" and "salience." Constraints arise from logical limits on the information that can be expressed in the representational notation, while salience arises from how easily the agent recovers the information (via perception) from the representational artifacts. Information that is recoverable from a representation is salient to the extent to which it is recoverable by automatic perceptual processing rather than through a controlled sequence of perceptual operators (Lohse, 1997, Zhang, 1997).

The discussion now turns to predictions based on differences between representational notations.

\section{Notations have ontological bias}

The first hypothesis claims that important guidance for collaborative learning discourse comes from ways in which a representational notation limits what can be represented (Reader, unpublished; Stenning \& Oberlander, 1995). A representational notation provides a set of primitive elements out of which representational artifacts are constructed. These primitive elements constitute an "ontology" of categories and structures for organizing the task domain. Learners will see their task in part as one of making acceptable representational artifacts out of these primitives. Thus, they will search for possible new instances of the primitive elements, and hence (according to this hypothesis) will be biased to think about the task domain in terms of the underlying ontology. Ontological bias will not be addressed further in this paper.

\section{Salient knowledge units are elaborated}

This hypothesis states that learners will be more likely to attend to, and hence elaborate on, the knowledge units that are perceptually salient in their shared representational workspace than those that are either not salient or for which a representational proxy has not been created. The 
To appear in Proc. 3rd Conference on Computer Supported Collaborative Learning, Stanford, December 11-15, 1999.

visual presence of the knowledge unit in the shared representational context serves as a reminder of its existence and any work that may need to be done with it. Also, it is easier to refer to a knowledge unit that has a visual manifestation, so learners will find it easier to express their subsequent thoughts about this unit than about those that require complex verbal descriptions (Clark \& Brennan, 1991). These claims apply to any visually shared representations. However, to the extent that two representational notations differ in kinds of knowledge units they make salient, these functions of reminding and ease of reference will encourage elaboration on different kinds of knowledge units.

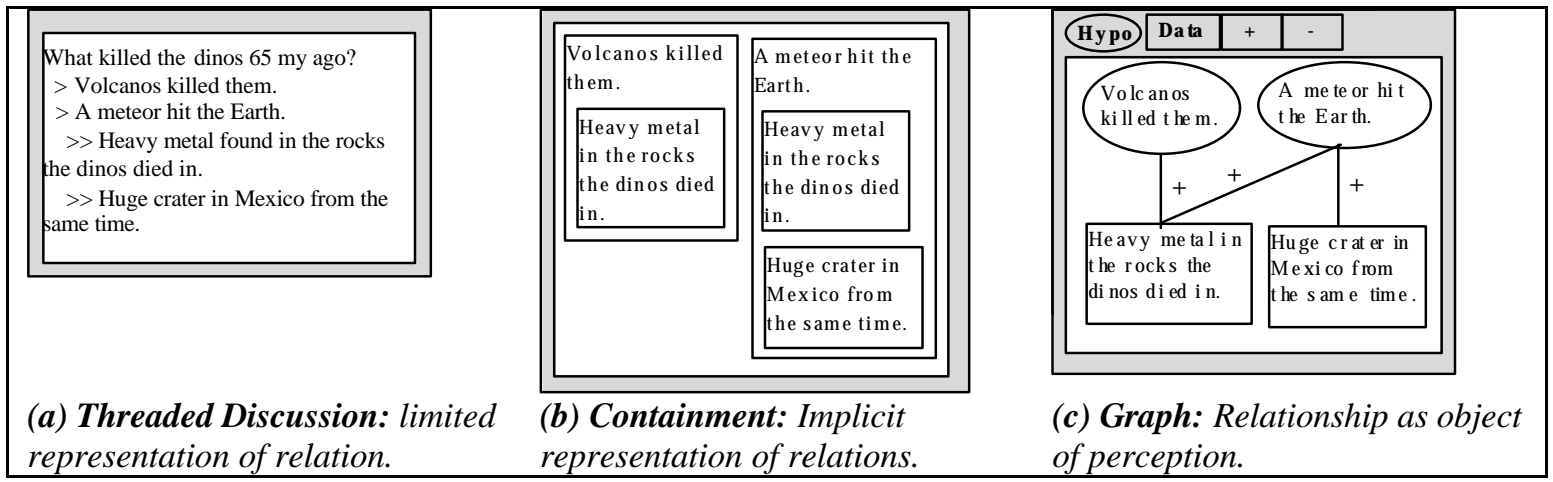

Figure 1. Example of Elaboration Hypothesis

For example, consider the three representations of a relationship between four statements shown in Figure 1. The relationship is one of evidential support. The middle notation uses an implicit device, containment, to represent evidential support, while the right-hand notation uses an explicit device, an arc. It becomes easier to perceive and refer to the relationship as an object in its own right as one moves from left to right in the figure. Hence the present hypothesis claims that relationships will receive more elaboration in the rightmost representational notation.

The opposite prediction is also plausible. Learners may see their task as one of putting knowledge units "in their place" in the representational environment. For example (according to this competing hypothesis), once a datum is placed in the appropriate hypothesis container (Figure 1b) or connected to a hypothesis (Figure1c), learners may feel it can be safely ignored as they move on to other units not yet placed or connected. Hence they will not elaborate on represented units. This suggests the importance of making missing information salient.

\section{Salience of missing units guides search}

Some representational notations provide structures for organizing knowledge units, in addition to primitives for construction of individual knowledge units. Unfilled "fields" in these organizing structures, if perceptually salient, can make missing knowledge units as salient as those that are present. If the representational notation provides structures with predetermined fields that need to be filled with knowledge units, the present hypothesis predicts that learners will try to fill these fields. 
To appear in Proc. 3rd Conference on Computer Supported Collaborative Learning, Stanford, December 11-15, 1999.

For example, Figure 2 shows artifacts from three notations that differ in salience of missing evidential relationships. In the textual representation, no particular relationships are salient as missing: no particular prediction about search for new knowledge units can be made. In the graph representation, the lack of connectivity of the volcanic hypothesis to the rest of the graph is salient. Hence this hypothesis predicts that learners will discuss its possible relationships to other statements. However, once some connection is made to the hypothesis, it will appear connected, so no further relationships will be sought. In the matrix representation, all undetermined relationships are salient as empty cells. The present hypothesis predicts that learners will be more likely to discuss many relationships between statements when using matrices.

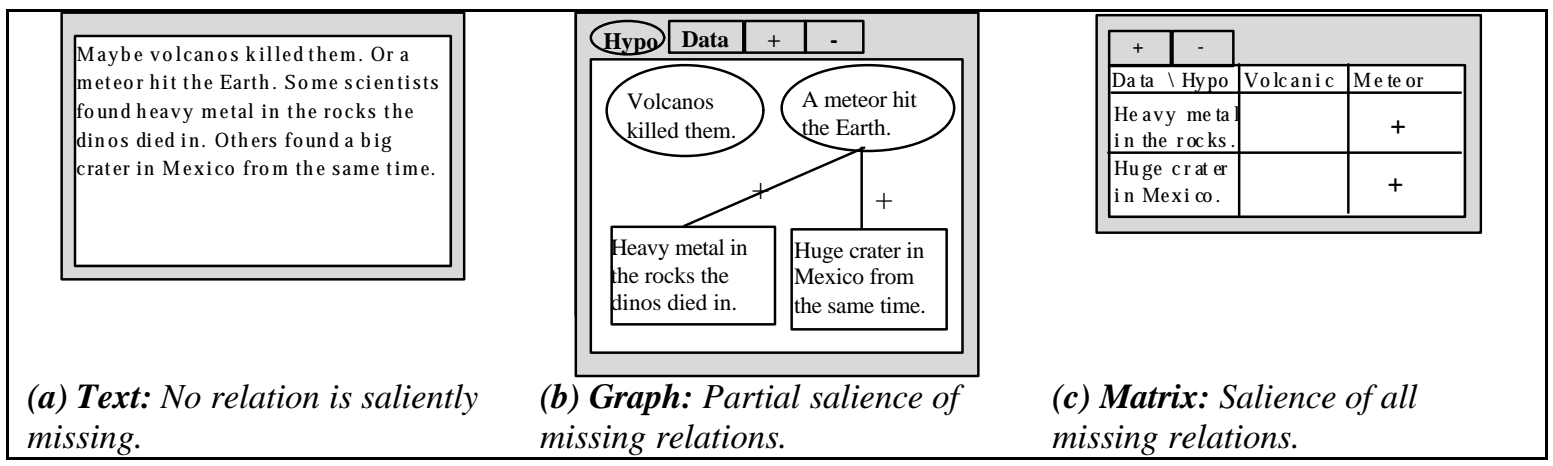

Figure 2. Example of Salient Absence Hypothesis

\section{Empirical studies}

The author has begun studies that test the effects of representational notations on collaborative discourse and learning. The question is not "what system is better?" but rather "what kinds of interactions, and therefore learning, does each representational notation encourage?" It may well be the case that all of the above representations are useful, albeit for different learning and problem solving phases or task domains.

The studies intentionally use representations that differ on more than one feature, as summarized in Table 1. The research strategy is to maximize the opportunity to observe predicted effects on learners' discourse, in order to explore the large space of experimental comparisons within the time scale on which collaborative technology is being adapted. These results will then inform well-motivated selection of studies that vary one feature at a time as needed to disambiguate alternate representational explanations for the results 
To appear in Proc. 3rd Conference on Computer Supported Collaborative Learning, Stanford, December 11-15, 1999.

\section{Table 1. Features of Selected Representational Formalisms}

\begin{tabular}{|l|l|l|l|l|}
\hline & Threaded & Containers & Graphs & Matrices \\
\hline $\begin{array}{l}\text { Organization } \\
\text { of Inquiry } \\
\text { Activity }\end{array}$ & $\begin{array}{l}\text { Discussion topics are } \\
\text { posted, followed by } \\
\text { chronologically } \\
\text { organized replies. }\end{array}$ & $\begin{array}{l}\text { Hypotheses are first } \\
\text { recorded as boxes in the } \\
\text { workspace. Empirical } \\
\text { observations are sorted } \\
\text { by placing them in } \\
\text { boxes. }\end{array}$ & $\begin{array}{l}\text { Hypotheses and } \\
\text { empirical observations } \\
\text { are recorded any time } \\
\text { as shapes placed in the } \\
\text { workspace. Evidential } \\
\text { relations are recorded } \\
\text { by linking shapes } \\
\text { together. }\end{array}$ & $\begin{array}{l}\text { Hypotheses and } \\
\text { empirical observations } \\
\text { are recorded at any time } \\
\text { column \& rows } \\
\text { (respectively) } \\
\text { Evidential relations are } \\
\text { recorded by placing } \\
\text { symbols in empty cells. }\end{array}$ \\
\hline $\begin{array}{l}\text { Ontology } \\
\text { implicit: } \\
\text { statements } \\
\text { reply chronology }\end{array}$ & $\begin{array}{l}\text { Implicit: } \\
\text { hypothesis } \\
\text { empirical } \\
\text { observation } \\
\text { consistency }\end{array}$ & $\begin{array}{l}\text { Explicit: } \\
\text { hypothesis } \\
\text { empirical } \\
\text { observation } \\
\text { consistency } \\
\text { inconsistency }\end{array}$ & $\begin{array}{l}\text { Explicit: } \\
\text { hypothesis } \\
\text { empirical } \\
\text { observation } \\
\text { consistency } \\
\text { inconsistency }\end{array}$ \\
\hline $\begin{array}{l}\text { Salience of } \\
\text { Malience of } \\
\text { Relations }\end{array}$ & $\begin{array}{l}\text { Implicit in Context: } \\
\text { reply chronology }\end{array}$ & $\begin{array}{l}\text { Implicit in Context: } \\
\text { consistency }\end{array}$ & $\begin{array}{l}\text { Explicit Object: } \\
\text { consistency } \\
\text { inconsistency }\end{array}$ & $\begin{array}{l}\text { Explicit Object: } \\
\text { consistency } \\
\text { inconsistency }\end{array}$ \\
\hline
\end{tabular}

\section{Experimental materials and procedure}

A pilot study was conducted comparing MS Word (unstructured text), MS Excel (tables), and Belvedere (graphs), with two pairs of subjects run in each condition. (Early results of the pilot are reported below.) Future experiments will use versions of Belvedere that have been modified to provide the alternative representations in Table 1 . This approach will reduce nonessential differences between the representational tools, and enable uniform recording of all manipulations of the representations in the Belvedere server database.

Subjects are presented with a "science challenge problem" in a web-browser. A science challenge problem presents a phenomenon to be explained (e.g., determining the cause of a mysterious disease), along with indices to relevant resources. It is important that these are relatively ill-structured problems: at any given point many possible knowledge units may reasonably be considered. This provides the necessary degrees of freedom within which representational bias can work.

One side of the computer screen contains the representational tool, such as Text, Containment, Graph, or Matrix. The other side contains a web browser open to the entry page for the science challenge materials. Students seated in front of the monitor are asked to read the problem statement in the web browser. They are then asked to identify hypotheses that provide candidate explanations of the phenomenon posed, and evaluate these hypotheses on the basis of laboratory studies and field reports obtained through the hypertext interface. They are asked to use the 
To appear in Proc. 3rd Conference on Computer Supported Collaborative Learning, Stanford, December 11-15, 1999.

representational tool to record the information they find and how it bears on the problem. The session is videotaped with the camera pointed at the screen over the shoulder of one of the participants. The camera is adjusted to show the screen in sufficient detail to see its contents, yet also show the immediate space around the screen to capture gestures in the vicinity of the screen. At the conclusion of the problem solving session, subjects are asked to write a brief essay and take a content knowledge test. Analysis is based on transcripts of subjects' spoken discourse, gestures, and modifications to the interface; as well as measures of learning outcomes (not discussed in this paper).

\section{Pilot study results}

The pilot data is currently under analysis. The purpose of this analysis is twofold: to identify trends suggesting that there is a phenomenon worthy of further study; and to refine analytic techniques. At this writing, pilot study videotapes from the six one-hour problem solving sessions have been transcribed and segmented, and limited coding has been completed. A segment was defined to be a gesture, a modification to the external representation, or a single speaker's turn in the dialogue, except that turns that expressed multiple propositions were broken into multiple segments. Segments were coded on the following dimensions (among others), using the QSR Nud*ist software package.

Representation: Values include Graph, Matrix, Text. This coding applies to an entire transcript, and indicates the independent variable for the session.

Mode: This dimension is used to filter and select segments for particular hypothesis tests. Values include Verbal, Gestural, and Representational (modifications to the software representations).

Evidential Content: Values include Consistent, Inconsistent, Choice. Identification of segments where subjects discuss or identify the nature of the evidential relationship between two statements as being one of consistency or inconsistency; or raise the question of which relationship holds.

A coarse-grained test of the Search hypothesis was conducted as follows. Recall that Search predicts that subjects will be more likely to seek evidential relations when using representations that prompt for these relations with empty structure (Text $<$ Graph $<$ Matrix). This analysis simply counted, for each treatment group, the percentage of verbal segments that were coded with any one of the three Evidential values (Consistent, Inconsistent, Choice). The results are shown in Table 2.

Examining the percentage of verbal segments that are concerned with evidence (rightmost column), the results appear to be consistent with the Search hypothesis (Text < Graph < Matrix). Although this trend is encouraging with respect to the question of whether there is a phenomenon worth investigating, this sample data cannot be taken as conclusive. Caveats (all of which are being addressed by ongoing work) include the small sample size (hence no test of significance), the lack of multiple coders (hence no test of inter-rater reliability), the need to test learning outcomes, and the need for a more direct test of the claim that representational state affects subsequent discourse processes. Analyses that are based on frequencies of utterances across the session as a whole fail to distinguish utterances seeking evidential relations from those 
To appear in Proc. 3rd Conference on Computer Supported Collaborative Learning, Stanford, December 11-15, 1999.

Table 2: Frequency Data for Evidential Statements

\begin{tabular}{|c|c|c|c|c|c|}
\hline Tool & Group & $\begin{array}{l}\text { Verbal } \\
\text { Segments }\end{array}$ & $\begin{array}{l}\text { Duration } \\
\text { (Sec) }\end{array}$ & $\begin{array}{l}\text { Evidence } \\
\text { Segments }\end{array}$ & \\
\hline \multirow[t]{3}{*}{ Text } & 5 & 200 & 2668 & 0 & $0 \%$ \\
\hline & 6 & 617 & 3706 & 13 & $2 \%$ \\
\hline & & 817 & 6374 & 13 & $2 \%$ \\
\hline \multirow[t]{3}{*}{ Graph } & 1 & 396 & 2801 & 18 & $5 \%$ \\
\hline & 2 & 227 & 1940 & 12 & $5 \%$ \\
\hline & & 623 & 4741 & 30 & $5 \%$ \\
\hline \multirow[t]{3}{*}{ Matrix } & 3 & 76 & 2526 & 1 & $1 \%$ \\
\hline & 4 & 550 & 2671 & 52 & $9 \%$ \\
\hline & & 626 & 5197 & 53 & $8 \%$ \\
\hline
\end{tabular}

elaborating on previous ones (i.e., between the Search and Elaborate hypotheses), or to show a causal relationship between the state of the representation and the subsequent discourse. A more sophisticated coding is required to test whether the representation or salient absence of a particular (kind of) knowledge unit influences search for or elaboration on that unit. This problem will be addressed as follows. Every change to the representations will be coded with the set of knowledge units that are (a) expressed or (b) saliently missing from that point onwards to the next change. Then, subsequent utterances within a time-window defined by a decay function will be tested for either (a) elaboration on those knowledge units or (b) search for other knowledge units related by evidential relations. This provides a more stringent test of the causal relationship between salience and discourse claimed by the research hypotheses.

\section{Qualitative observations}

Examples and discussion of the artifacts created and of transcripts are provided here to help illustrate the predicted effects and related issues.

The document created by group 5 (text) had no expression of evidential relations between the hypotheses and data, and there was no overt discussion of evidential relations in the transcript of verbal discourse. All of the discussion of evidence in the text condition occurred in group 6 at the end of their session (the longest session in the pilot study), when the subjects spontaneously identified a hypothesis impacted by each datum gathered.

A document produced by group 1 (graph) is reproduced in Figure 3, followed by a portion of the corresponding transcript in Table 3 . Note the linearity of the graph (normally considered a nonlinear medium). The pattern of \{identify, categorize, add, link\} seen in the transcript (underlined) is typical of interactions in this transcript. This pattern of activity, which leads to the linearity of the graph, is consistent with the competitor to the Elaboration hypothesis: subjects may feel that the primary task is to connect each new statement to something else, after which it can be ignored. 
To appear in Proc. 3rd Conference on Computer Supported Collaborative Learning, Stanford, December 11-15, 1999.

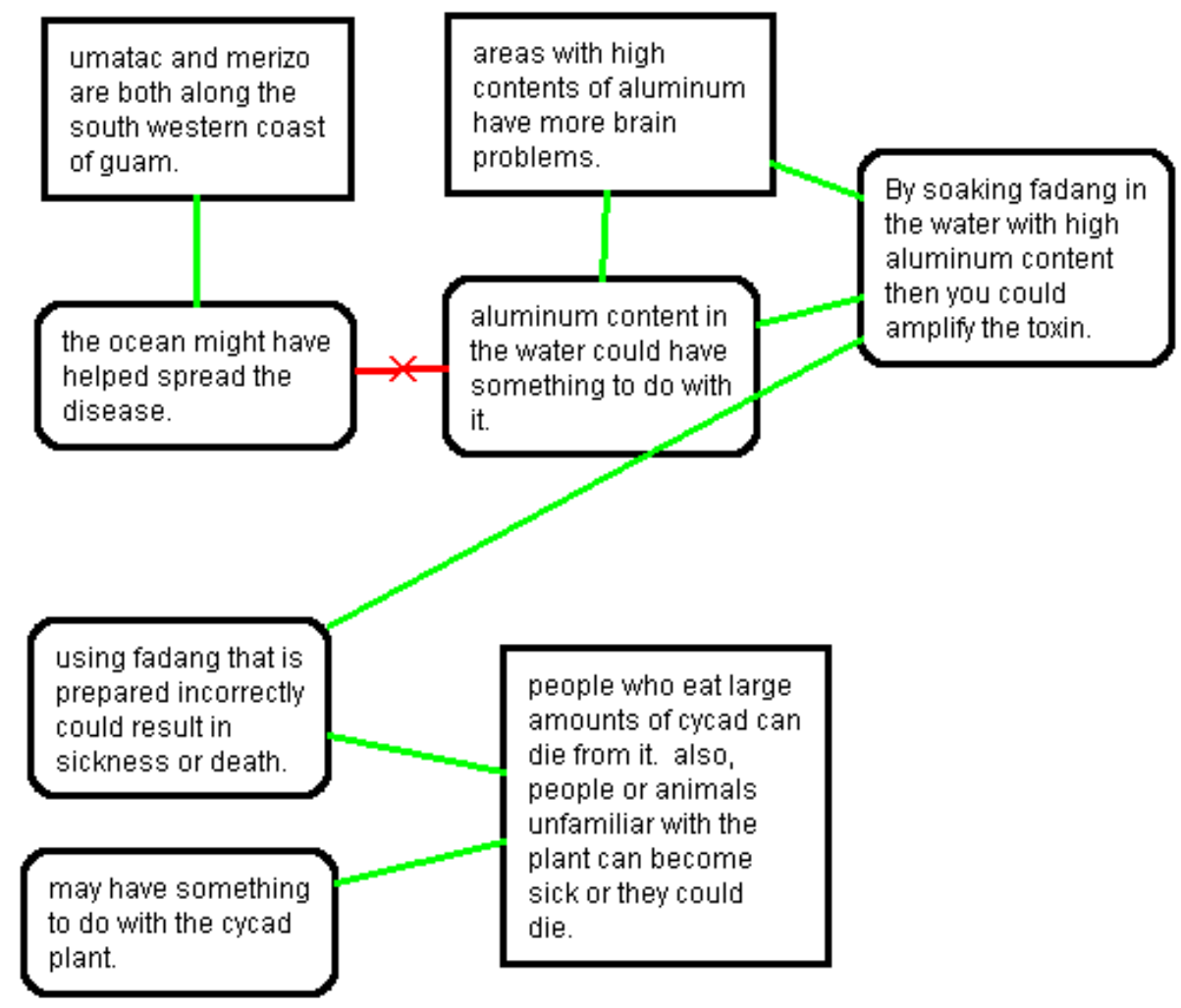

Figure 3. Group 1's Graph

Table 3. Group 1's Transcript Sample

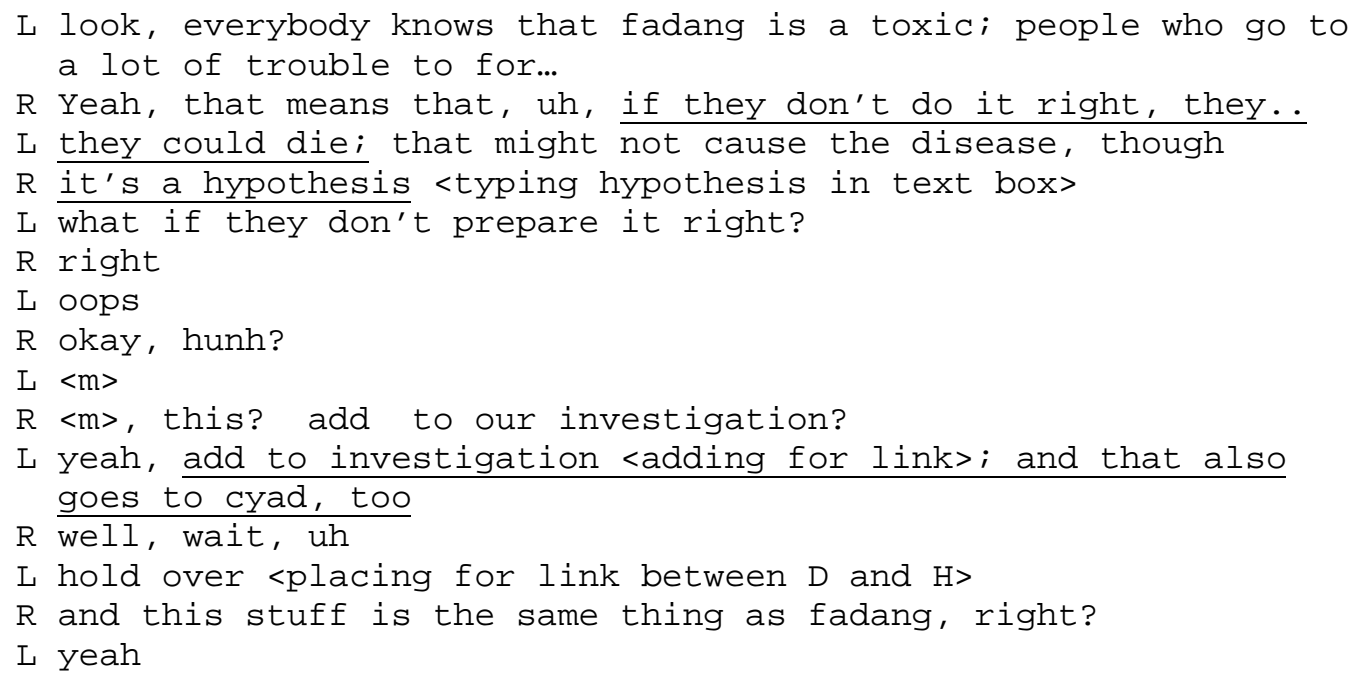


To appear in Proc. 3rd Conference on Computer Supported Collaborative Learning, Stanford, December 11-15, 1999.

Table 4. Group 4's Matrix

\begin{tabular}{|c|c|c|c|c|c|}
\hline Data & $\begin{array}{l}\text { food poisoning } \\
\text { found in Central } \\
\text { America-FADANG }\end{array}$ & $\begin{array}{l}\text { radiation from } \\
\text { Hiroshima/Nagaso } \\
\text { ki in the Pacific }\end{array}$ & $\begin{array}{l}\text { diet in The south } \\
\text { Pacific }\end{array}$ & $\begin{array}{l}\text { nerve tangles found } \\
\text { in spinal cords } \\
\text { causing desease, } \\
\text { similar to } \\
\text { Alziemer's }\end{array}$ & $\begin{array}{l}\text { Radiation } \\
\text { temporarily } \\
\text { contaminated the } \\
\text { food of the South } \\
\text { pacific }\end{array}$ \\
\hline $\begin{array}{l}\text { nutritionist claims that } \\
\text { chamorros, found in } \\
\text { FADANG, killed several } \\
\text { people }\end{array}$ & confirm & conflict & confirm & conflict & confirms \\
\hline $\begin{array}{l}\text { Nutritionist claims that } \\
\text { lack of Calcium in } \\
\text { Pacific region causes } \\
\text { brain desease }\end{array}$ & conflict & conflict & confirm & conflict & conflicts \\
\hline $\begin{array}{l}\text { spinal/brain } \\
\text { disfunctions causing } \\
\text { desease }\end{array}$ & conflict & conflict & conflict & confirm & conflicts \\
\hline $\begin{array}{l}\text { Certain Amino Acids } \\
\text { found in food (peas) } \\
\text { cause disfunction in the } \\
\text { brain }\end{array}$ & confirms & conflicts & confirms & confirms & confirms \\
\hline $\begin{array}{l}\text { Test between 1941-1945 } \\
\text { proved that all patients } \\
\text { in the hospital were } \\
\text { Chamorro and had } \\
\text { ALS }\end{array}$ & confirms & conflicts & confirms & confirms & confirms \\
\hline $\begin{array}{l}\text { The same test run now } \\
\text { proved that there's a } \\
\text { major decline in the rate } \\
\text { of Als found in } \\
\text { Guamian }\end{array}$ & confirms & confirms & conflicts & confirms & confirm \\
\hline
\end{tabular}

\section{Table 5. Group 4's Transcript Sample}

R Found in chickling peas. Chickling peas...

L Peas. All right. <points to screen> Now. What hypothesis is

this? Food poisoning. Yeah. Okay. Go down. Let's see. Supports.

$\mathrm{R}$ Confirms. Confirm. You might want to use the same word you're using the whole time.

L Is there any chance we could use this for the science fair? No. It conflicts.

R Diet in South Pacific, yes?

L No, no, no, no, no. <points to screen>

$\mathrm{R}$ Conflicts and then confirms.

L Conflicts. Is it. Confirms. What is this one? Oh, the brain.

R Well, yeah.

L Yeah, yeah. Okay. Confirms. Well, I think now we should, like, rule out this <points to hypothesis in table> one because it has had nothing but conflicts. The Hiroshima ...that we thought of ourself.

$R$ It conflicts, conflicts, conflicts. It hasn't had one, uh... 
To appear in Proc. 3rd Conference on Computer Supported Collaborative Learning, Stanford, December 11-15, 1999.

Finally, a matrix produced by group 4 (Excel) is reproduced in Table 4, followed by a portion of the group's transcript in Table 5. Table 4 is especially striking because students were not specifically instructed to fill in all the cells. In the transcript, note the systematic identification of evidential relations as students work down the column, and the appropriate use of the column to rule out a hypothesis that the students proposed (radiation from atomic bombs caused disease, second column).

\section{Summary}

Prior experience with Belvedere suggested that variation in features of the representational tools can have a significant effect on the learners' knowledge-building discourse and on learning outcomes. The paper sketched a theoretical analysis of the role of constraints and salience in representational bias, outlined an investigation being undertaken by the author, and reported promising but preliminary results of a pilot study. Continued work in this area will inform the design of future software learning environments and provide a better theoretical understanding of the role of representational bias in guiding learning processes.

\section{Acknowledgments}

This work was funded by DoDEA's Presidential Technology Initiative while the author was affiliated with the University of Pittsburgh's Learning Research and Development Center, and by NSF's Learning and Intelligent Systems program under the author's present affiliation. The author is grateful to numerous LRDC colleagues, especially Alan Lesgold, Eva Toth, and Arlene Weiner, for collaborations on the design of Belvedere; to Micki Chi, Martha Crosby, and John Levine for discussions concerning the role of representations in learning, visual search, and social aspects of learning, respectively; and to Cynthia Liefeld and David Pautler for assistance with the pilot studies.

\section{Bibliography}

Bell, P. (1997). Using argument representations to make thinking visible for individuals and groups. In Proc. Computer Supported Collaborative Learning '97, pp. 10-19. University of Toronto, December 10-14, 1997.

Clark, H.H. \& Brennan, S.E. (1991). Grounding in Communication. In L.B. Resnick, J.M. Levine and S.D. Teasley (eds.), Perspectives on Socially Shared Cognition, American Psychological Association, 1991, pp. 127-149.

Conklin, J. \& Begeman, M.L. (1987). gIBIS: A hypertext tool for team design deliberation. In Hypertext'97 Proceedings, Chapel Hill, NC, pp 247-252. New York: ACM.

Guzdial, M. (1997). Information ecology of collaborations in educational settings: Influence of tool. Proc. 2nd Int. Conf. on Computer Supported Collaborative Learning (CSCL'97), Toronto, December 10-14, 1997. pp. 91-100. 
To appear in Proc. 3rd Conference on Computer Supported Collaborative Learning, Stanford, December 11-15, 1999.

Guzdial, M., Hmelo, C., Hubscher, R., Nagel, K., Newstetter, W., Puntambekar, S., Shabo, A., Turns, J., \& Kolodner, J. L. (1997). Integrating and guiding collaboration: Lessons learned in Computer-Supported Collaborative Learning research at Georgia Tech. Proc. 2nd Int. Conf. on Computer Supported Collaborative Learning (CSCL'97), Toronto, December 10-14, 1997. pp. 91-100.

Koedinger, K. (1991). On the design of novel notations and actions to facilitate thinking and learning. Proc. Int. Conference on the Learning Sciences, pp. 266-273. Charlottesville, VA: Association for the Advancement of Computing in Education. 1991.

Kotovsky, K. and H. A. Simon (1990). "What makes some problems really hard: Explorations in the problem space of difficulty." Cognitive Psychology 22: 143-183.

Larkin, J. H. \& Simon, H. A. (1987). Why a diagram is (sometimes) worth ten thousand words. Cognitive Science 11(1): 65-99. 1987.

Lohse, G. L. (1997). Models of graphical perception. In M. Helander, T. K. Landauer, \& P. Prabhu (Eds.), Handbook of Human-Computer Interaction (pp. 107-135). Amsterdam: Elsevier Science B.V.

McGuiness, C. (1986). "Problem representation: The effects of spatial arrays.” Memory \& Cognition 14(3): 270-280.

Okada, T. \& Simon, H. A. (1997). Collaborative discovery in a scientific domain. Cognitive Science 21(2): 109-146.

O'Neill, D. K., \& Gomez, L. M. (1994).The collaboratory notebook: A distributed knowledgebuilding environment for project-enhanced learning. In Proc. Ed-Media '94, Vancouver, BC.

Perkins, D.N. (1993). Person-plus: A distributed view of thinking and learning. In G. Salomon (Ed). Distributed cognitions: Psychological and Educational Considerations pp. 88-

111.Cambridge: Cambridge University Press.

Puntambekar, S., Nagel, K., Hübscher, R., Guzdial, M., \& Kolodner, J. (1997). Intra-group and Intergroup: An exploration of Learning with Complementary Collaboration Tools. In Proc Computer Supported Collaborative Learning Conference '97, pp. 207-214. University of Toronto, December 10-14, 1997.

Ranney, M., Schank, P., \& Diehl, C. (1995). Competence versus performance in critical reasoning: Reducing the gap by using Convince Me. Psychology Teaching Review, 1995, 4(2).

Reader, W. (Unpublished). Structuring Argument: The Role of Constraint in the Explication of Scientific Argument, manuscript dated November 1997. 
To appear in Proc. 3rd Conference on Computer Supported Collaborative Learning, Stanford, December 11-15, 1999.

Roschelle, J. (1994). Designing for Cognitive Communication: Epistemic Fidelity or Mediating Collaborative Inquiry? The Arachnet Electronic Journal on Virtual Culture, May 16, 1994. Available: ftp://ftp.lib.ncsu.edu/pub/stacks/aejvc/aejvc-v2n02-roschelle-designing

Scardamalia, M., Bereiter, C., Brett, C., Burtis, P.J., Calhoun, C., \& Smith Lea, N. (1992). Educational applications of a networked communal database. Interactive Learning Environments, 2(1), 45-71.

Smolensky, P., Fox, B., King, R., \& Lewis, C. (1987). Computer-aided reasoned discourse, or, how to argue with a computer. In R. Guindon (Ed.), Cognitive science and its applications for human-computer interaction (pp. 109-162). Hillsdale, NJ: Erlbaum.

Stenning, K. \& Oberlander, J. (1995). A cognitive theory of grahical and linguistic reasoning: Logic and implementation. Cognitive Science 19(1): 97-140. 1995.

Suthers, D. (1995). Designing for internal vs. external discourse in groupware for developing critical discussion skills. CHI'95 Research Symposium. Denver, May 1995.

Suthers, D., Toth, E., and Weiner, A. (1997). An Integrated Approach to Implementing Collaborative Inquiry in the Classroom. Proc. 2nd Int. Conf. on Computer Supported Collaborative Learning (CSCL'97), Toronto, December 10-14, 1997. pp. 272-279.

Suthers, D. and Weiner, A. (1995). Groupware for developing critical discussion skills. CSCL '95, Computer Supported Cooperative Learning, Bloomington, Indiana, October 17-20, 1995.

Utgoff, P. (1986). Shift of bias for inductive concept learning. In R. Michalski, J. Carbonell, T. Mitchell (Eds.) Machine Learning: An Artitificial Intelligence Approach, Volume II, Los Altos: Morgan Kaufmann 1986, pp. 107-148.

Wan, D., \& Johnson, P. M. (1994). Experiences with CLARE: a Computer-Supported Collaborative Learning Environment. International Journal of Human-Computer Studies, October, 1994.

Wojahn, P. G., Neuwirth, C. M., \& Bullock, B. (1998). Effects of interfaces for annotation on communication in a collaborative task. Conf. on Human Factors in Computing Systems (CHI 98), 18-23 April, Los Angeles, pp. 456-463.

Zhang, J. (1997). The nature of external representations in problem solving. Cognitive Science, 21(2): 179-217, 1997.

\section{Author's address}

Daniel D. Suthers (suthers@ hawaii.edu)

University of Hawai'i at Manoa, Dept. of Information and Computer Sciences, 1680 East West Road, Honolulu, HI 96822. Tel. (808) 956-3890. Fax (808) 956-9639. 